\title{
Fibroblast Heterogeneity
}

\section{Existence of Functionally Distinct Thy $1^{+}$and Thy $1^{-}$Human Female Reproductive Tract Fibroblasts}

\author{
Laura Koumas, ${ }^{*}$ Anne E. King, ${ }^{\dagger}$ \\ Hilary O. D. Critchley, ${ }^{\dagger}$ Rodney W. Kelly, ${ }^{\dagger}$ and \\ Richard P. Phipps* \\ From the Department of Microbiology and Immunology,* Cancer \\ Center, University of Rochester School of Medicine and Dentistry, \\ Rochester, New York; and the Medical Research Council, ${ }^{\dagger}$ \\ Reproductive Biology Unit, Center for Reproductive Biology, \\ Edinburgh, Scotland
}

Little is known about fibroblasts from the female reproductive tract, much less whether or not functional subsets exist. Fibroblasts are key as sentinel cells for recruiting white blood cells and for wound healing. The purpose of this research was to evaluate the possibility that functional subsets of fibroblasts exist in the human female reproductive tract. The strategy used was to define fibroblast subpopulations based on their surface expression of the Thy 1 antigen. In situ staining of human myometrium and endometrium showed heterogeneous staining for Thy 1. Freshly derived strains of fibroblasts from the myometrium and endometrium also demonstrated heterogeneous Thy 1 expression. For the first time, using magnetic beading and fluorescence-activated cell sorting, human myometrial fibroblasts were successfully separated into functionally unique Thy $1^{+}$and Thy $1^{-}$ subsets. Both subsets produced the proinflammatory cytokines interleukin (IL)-6 and IL-8 after IL-1 $\beta$ stimulation, but only the Thy $1^{+}$subset produced MCP-1. Furthermore, only Thy $1^{+}$fibroblasts up-regulated CD40 surface expression with IL-1 $\beta$ or interferon- $\gamma$ treatment. Engagement of CD40 in the Thy $1^{+}$subpopulation induced IL-6, IL-8, and MCP-1. The discovery of functional subsets of reproductive tract fibroblasts now permits assessment of their roles in the normal functions of the reproductive tract and in disease states such as adhesions and menorrhagia. (Am J Patbol 2001, 159:925-935)

The human female reproductive tract is a complex set of tissues whose roles are crucial for human existence. The maintenance of pregnancy and the initiation of labor are dependent on how these tissues respond to local stimuli, such as sex steroid hormones, cytokines, and lipid mediators. Furthermore, the endometrium must repeatedly differentiate and regenerate in response to the aforementioned mediators every menstrual cycle. A controlled and well-timed inflammatory response must also occur as part of menstruation, host defense, and cervical ripening during labor. ${ }^{1,2}$ An exuberant or inappropriate inflammatory response could lead to pathophysiological situations such as preterm labor and menstrual dysfunction. Premature labor afflicts $7 \%$ of women, whereas $20 \%$ of women suffer from menstrual dysfunction.

CD40 is a 45-to 50-kd type I glycoprotein and a member of the tumor necrosis factor- $\alpha$ receptor family. CD40 was initially discovered on the surface of B lymphocytes ${ }^{3}$ and was later shown to be expressed on several cell types including monocytes, vascular endothelial cells, ${ }^{4}$ and some epithelial cells. ${ }^{5}$ CD40 is the cognate receptor for CD40 ligand (CD40L/CD154), which is found on activated T cells, mast cells, ${ }^{6}$ eosinophils, ${ }^{7}$ and basophils. ${ }^{6}$ Platelets were recently found to have an intracellular pool of preformed CD40L, which is surface-expressed on activation. $^{8}$ The CD40-CD40L interaction is a critical activation signal in hematopoietic cells, but is also important in nonhematopoietic cell activation. Human fibroblasts from several sites were recently shown to express CD40 and to up-regulate its expression after interferon (IFN)- $\gamma$ treatment. ${ }^{9,10}$ CD40 ligation on some types of fibroblasts results in the production of proinflammatory cytokines such as interleukin (IL)-6 and IL-8. 9,11,12 Fibroblasts from the endometrium, myometrium, and cervix not only express CD40, but also up-regulate synthesis of the chemoattractant MCP-1 in addition to IL-6 and IL-8 after CD40 engagement with CD40L. ${ }^{13}$

An emerging paradigm is that fibroblasts are not mere structural cells, but have key functional roles in the tissue in which they reside. The concept of fibroblasts as sentinel cells has provided a role for fibroblasts as initiators of inflammation. ${ }^{14,15}$ Fibroblasts can produce proinflammatory cytokines and prostaglandins on stimulation and can thus form a bridge of communication with classic

Supported by United States Public Health Service (grants DE11390, HL56002, EY08976, ES01247), the Burroughs-Wellcome Foundation, and the Pepper Center.

Accepted for publication May 14, 2001

Address reprint requests to Richard P. Phipps, University of Rochester, Cancer Center, Box 704, 601 Elmwood Ave., Rochester, NY 14642. E-mail: richard_phipps@urmc.rochester.edu. 
immune cells. Fibroblasts are also the major tissue structural cell and source of collagen. It is the collagen matrix that gets degraded by infiltrating neutrophils and prepares the human cervix (ie, cervical softening or ripening) for parturition. ${ }^{16}$

Besides their role as sentinel cells, much interest lies in the concept that, like lymphocytes, fibroblasts are heterogeneous and that functional subpopulations exist. Fibroblasts differ between organs and also within tissues. Evidence supporting fibroblast heterogeneity includes differences in morphology, ${ }^{17}$ proliferation rates, ${ }^{18}$ collagen synthesis, ${ }^{19} \mathrm{PGE}_{2}$ synthesis, ${ }^{20}$ and effects of estrogen. ${ }^{21}$ To successfully define fibroblast subsets, the surface glycophosphatidylinositol-linked protein Thy 1 was previously chosen by our laboratory as the discriminatory cell surface marker. In these studies, we demonstrated that mouse lung and spleen fibroblasts were separable into Thy $1^{+}$and Thy $1^{-}$subpopulations that defined functional differences. ${ }^{17,22,23}$ Separation of fibroblasts according to expression of Thy 1 and characterization of subsets' distinct roles will lead to advances in understanding fibroblast biology and is key for understanding normal and abnormal wound healing and for defining the role of fibroblasts in inflammation.

We investigated the possibility that functional subpopulations of fibroblasts exist in the human female reproductive tract. These surface marker studies based on Thy 1 are the first we are aware of that clearly demonstrate fibroblast heterogeneity in the human female reproductive tract (myometrium and endometrium). To demonstrate proof of concept, myometrial fibroblasts were separated into Thy $1^{+}$ and Thy $1^{-}$fractions and showed unique functional differences. The identification of fibroblasts from the reproductive tract that have unique profiles suggests the importance of considering fibroblast subpopulations in development and dysfunctions of the female reproductive tract.

\section{Materials and Methods}

\section{Tissue Collection}

Tissue samples were obtained as described in King and colleagues. ${ }^{13}$ In brief, biopsies were collected from the myometrium and endometrium of women that underwent gynecological procedures for benign conditions. All women had regular menstrual cycles (25 to 35 days) and did not receive any form of hormonal treatment in the 3 months before the procedure. Donors were between the ages of 20 to 40 years and were premenopausal. Endometrial biopsies were dated according to the criteria of Noyes and colleagues ${ }^{24}$ and circulating sex steroid levels were consistent with the date of the last menstrual period. Written consent was received from all patients and ethical approval for biopsy collection was received from Lothian Research Ethics Committee.

\section{Fibroblast Strain Derivation}

Myometrial or endometrial tissue was cut into pieces of $1-\mathrm{mm}^{3}$ approximate size and placed under glass cover- slips in 100-mm tissue culture dishes (Costar, Cambridge, MA). This resulted in fibroblast outgrowth from the tissue as previously described. ${ }^{10,12}$ The cells were stained for fibroblast markers using immunostaining. Cells were positive for vimentin (stains fibroblasts), but negative for cytokeratin (epithelial cell marker), $\alpha$-smooth muscle actin (myofibroblasts and smooth muscle cells), CD34 (endothelial cell marker), and CD45 (bone marrowderived cell marker). The cells that proliferate under these conditions had fibroblast-consistent morphology. Fibroblasts used for separation into subsets and for cytokine analysis were as early passage as possible. Endometrial and myometrial strains were cultured in RPMI 1640 media (Life Technologies, Gaithersburg, MD) supplemented with 10\% fetal bovine serum (FBS; Hyclone Laboratories, Logan, UT), nonessential amino acids, and gentamicin (Life Technologies). No additional factors were included in the RPMI 1640 culture media. Fibroblasts were removed from confluent cultures by dissociating monolayers with $1: 1 \quad 0.05 \%$ Trypsin:0.1\% ethylenediaminetetraacetic acid solution (Life Technologies), and $5 \times 10^{5}$ cells were reseeded in $75-\mathrm{mm}^{2}$ tissue culture flasks (Costar). Cultures were grown 3 to 4 days before trypsinization.

\section{Immunohistochemistry}

Immunohistochemistry for Thy 1 expression was done on endometrial and myometrial tissue sections. Biopsies for immunohistochemistry were collected with an endometrial suction curette (Pipelle, Laboratoire CCD, Paris, France) and full-thickness endometrial samples were also obtained. These included superficial and basal endometrium along with the endometrial-myometrial junction. Frozen tissue sections were fixed in neutral-buffered formalin for 10 minutes at room temperature. Endogenous peroxidase activity was blocked with $3 \%$ hydrogen peroxide and blocked with diluted normal horse serum (Vector Laboratories, Burlingame, CA) for 20 minutes at room temperature. Tissue sections were incubated overnight at $4^{\circ} \mathrm{C}$ with mouse monoclonal anti-human Thy 1 (F15-421-5, $10 \mu \mathrm{g} / \mathrm{ml}$ ), a cell-free supernatant that was prepared from a mouse hybridoma. An isotype control antibody mouse IgG1 (Caltag, Burlingame, CA) was included at $10 \mu \mathrm{g} / \mathrm{ml}$. Sections were then incubated with biotinylated horse anti-mouse IgG (Vector Laboratories) and the avidin-biotin-peroxidase detection system was used (Elite ABC 6101; Vector Laboratories). Sections were counterstained with hematoxylin.

\section{Immunofluorescence Analysis and Fibroblast Subset Separation}

Immunofluorescence was used to determine Thy 1 expression in the myometrial and endometrial fibroblast strains. Monolayers were dissociated by trypsin:ethylenediaminetetraacetic acid solution and suspended in PAB (phosphate-buffered saline containing 1\% bovine serum albumin and $0.1 \%$ sodium azide). It was previously shown that Thy 1 does not get cleaved from the surface 
of fibroblasts with trypsin treatment. ${ }^{17,25,26}$ As a primary anti-human Thy 1 antibody, a cell-free supernatant was used that was prepared from a mouse hybridoma (F15421-5). One million cells were used for each sample. Cell samples were incubated with F15-421-5 or an isotype control antibody for 45 minutes at $4^{\circ} \mathrm{C}$. Samples were washed three times with $\mathrm{PAB}$ and then incubated with goat anti-mouse immunoglobulin conjugated with fluorescein isothiocyanate (Sigma Chemical Co., St. Louis, MO) as a secondary antibody for 45 minutes at $4^{\circ} \mathrm{C}$. Cells were then washed again three times and resuspended in $1 \mathrm{ml} \mathrm{PAB}$ and analyzed on a FACSCalibur flow cytometer (Sigma Chemical Co.). CellQuest software was used for data analysis. The above procedure was also used to stain fibroblasts for confocal microscope analysis.

Fibroblast subset separation was accomplished by first sorting by fluorescence-activated cell sorting according to Thy 1 expression followed by three to four rounds of magnetic bead selection. Magnetic beads (Dynal, Oslo, Norway) had a human anti-mouse monoclonal antibody attached onto them via a DNA linker. Cells were stained for Thy 1 with F15-421-5 and incubated with the beads for 30 minutes at $4^{\circ} \mathrm{C}$. By negative selection, the Thy $1^{-}$population was isolated using a magnetic device to hold the beads. After incubation with DNase for 15 minutes, the Thy $1^{+}$population was released and isolated. Once fibroblasts were separated into subsets, their Thy 1 phenotype remained stable over culture. Early passage cells were used throughout experiments (passages 4 to 12). Furthermore, fibroblast subsets were tested for Thy 1 expression by flow cytometry before each experiment to ensure that Thy $1^{+}$and Thy $1^{-}$profiles were maintained. A cytogenetic assessment of ploidy was also performed in both subsets by propidium iodide staining in concert with flow cytometry. The ploidy was normal and indicated stable DNA content (data not shown).

\section{CD40 Expression by Flow Cytometry}

Fibroblasts were cultured with or without IFN- $\gamma(500 \mathrm{U} / \mathrm{ml})$ for up to 72 hours or $1 \mathrm{~L}-1 \beta(10 \mathrm{ng} / \mathrm{ml})$ for 24 hours. The cells were lightly trypsinized, washed, and resuspended in PAB. One million cells were used for each sample. Mouse anti-human CD40 (G28-5) was used as the primary antibody for 45 minutes at $4^{\circ} \mathrm{C}$. Mouse IgG1 (Sigma Chemical Co.) was used as the isotype control. The cells were washed three times with PAB and then incubated with goat anti-mouse immunoglobulin conjugated with fluorescein isothiocyanate (Sigma Chemical Co.) as a secondary antibody for 45 minutes at $4^{\circ} \mathrm{C}$. Cells were then washed again three times and resuspended in $1 \mathrm{ml}$ of PAB and analyzed on a FACSCalibur.

\section{Cytokine Production}

Fibroblasts were seeded at $5 \times 10^{4}$ cells/well in a 48-well plate (Costar) in RPMI 1640, 10\% FBS supplemented with gentamicin. After 24 hours, cells were serum-starved for 72 hours in fresh media containing $0.5 \%$ FBS with or without IFN- $\gamma(500 \mathrm{U} / \mathrm{ml})$. The culture supernatant was then removed again and serum-free media was added. The treatments used for the final 24 hours were no stimulation, IFN- $\gamma$ or IL-1 $\beta$ (10 ng/ml; R\&D Systems Inc., Minneapolis, MN) treatments. Finally, supernatants were harvested and tested for IL-6, IL-8, and MCP-1 production by enzyme-linked immunosorbent assay. ${ }^{27}$

CD40L was generated from insect cell membranes expressing CD40L as described previously. ${ }^{11,12}$ CD40 ligation was accomplished by addition of CD4OL when cells were serum-free for the final 24 hours. The antiCD40L antibody MK13A4 (2.5 $\mathrm{gg} / \mathrm{ml}$; Boehringer Ingelheim) was used the final 24 hours on fibroblasts stimulated with IFN- $\gamma$ and CD40L. Mouse IgG1 (Sigma Chemical Co.) was also added at $2.5 \mu \mathrm{g} / \mathrm{ml}$ as a control antibody.

Statistical analysis was done using the student's paired two-tailed $t$-test. A probability of $P<0.05$ (assigned as ${ }^{*}$ ) or $P<0.01$ (assigned as ${ }^{*}$ ) indicates statistical significance.

\section{Results}

\section{In Situ Expression of Thy 1 in Endometrium and Myometrium and Development of Fibroblast Strains Heterogeneous for Thy 1 Expression}

In situ staining of human myometrial tissue sections for Thy 1 , revealed Thy 1 expression only in particular regions (Figure 1). Thy 1 immunoreactivity was present in smooth muscle cells and in some fibroblastic stromal cells of the myometrium (Figure 1a). Staining of endometrial tissue showed Thy 1 expression to be scattered among the fibroblastic stromal cells and to be highly expressed in some endothelial cells and concentrated in the layers of structural cells surrounding the spiral arterioles (Figure 1, $\mathrm{c}$ and $\mathrm{e}$ ). There is not a significant difference in Thy 1 staining throughout the menstrual cycle based on the samples herein evaluated (data not shown). In the tissue sections tested, Thy 1 is highly expressed around the majority of the blood vessels (data not shown). As shown in Figure 1c, Thy 1 is mainly localized to the perivascular region of the endometrium basalis. It was interesting to observe a different staining pattern for Thy 1 in the endometrium functionalis (Figure 1e), where Thy 1 expression is widely distributed among fibroblastic stromal cells. Thus the endometrial basalis region shows Thy 1 to be localized around blood vessels (Figure 1c), whereas in the functionalis region Thy 1 is expressed throughout the stroma (Figure 1e) as well as around blood vessels (data not shown). Besides staining individual cells, Thy 1 may also be localized extracellularly (Figure 1, a and c). Thy 1 has recently been shown to exist in soluble form in serum. ${ }^{28}$ The presence of soluble Thy 1 could reflect the contiguous staining of Thy 1 in the myometrium and endometrium. 

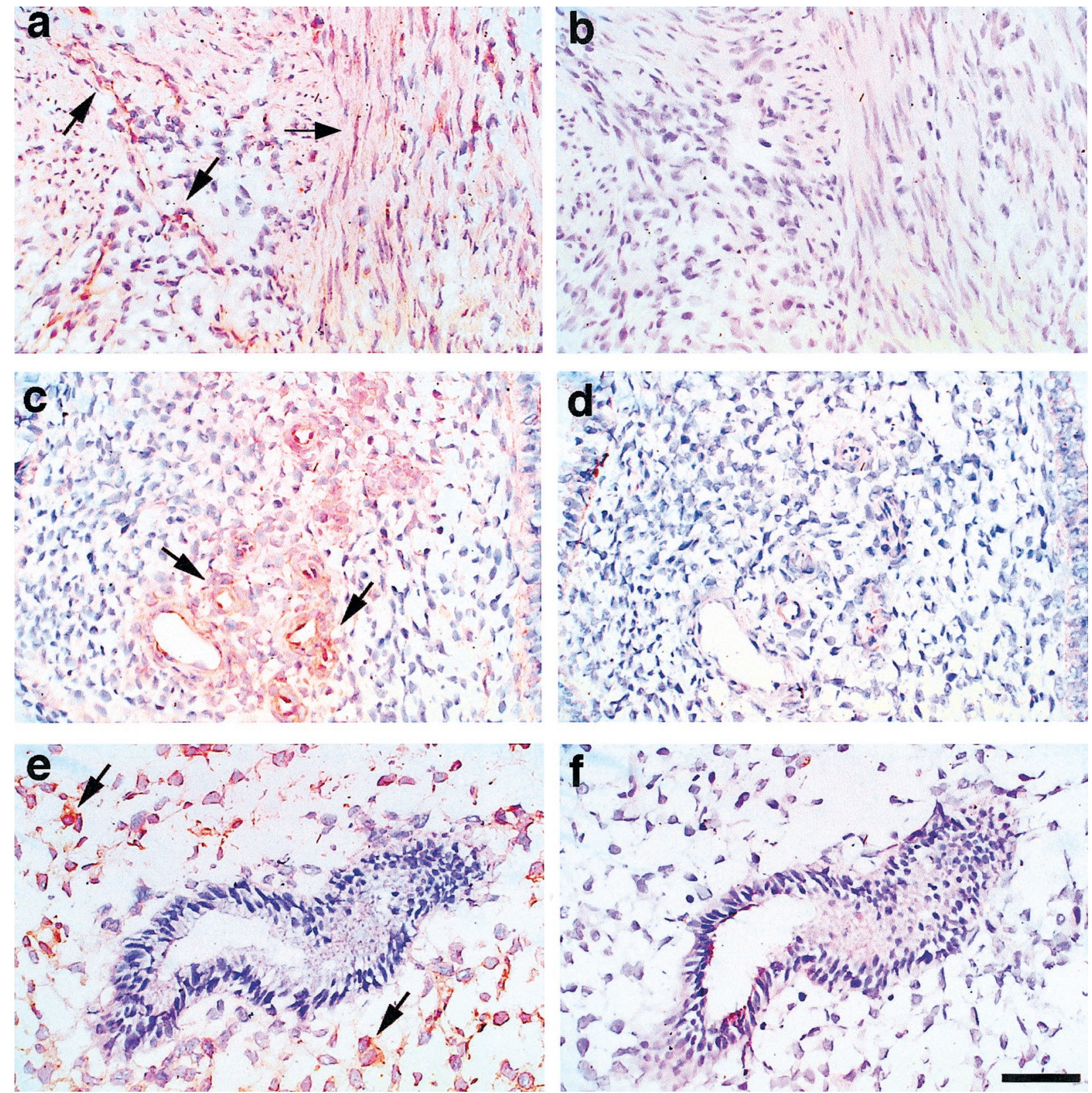

Figure 1. Immunohistochemical localization of Thy 1 in human myometrium and endometrium. Frozen tissue sections were fixed in formalin and blocked with $5 \%$ normal horse serum for 20 minutes at room temperature. Tissue sections were incubated overnight at $4^{\circ} \mathrm{C}$ with mouse monoclonal anti-human Thy 1 $(\mathrm{F} 15-421-5,10 \mu \mathrm{g} / \mathrm{ml})$. A negative control antibody (mIgG1) was included at $10 \mu \mathrm{g} / \mathrm{ml}$. Sections were incubated with biotinylated horse anti-mouse IgG and the avidin-biotin-peroxidase detection system was used. Sections were counterstained with hematoxylin. a: Myometrium. Immunoreactivity is present in some smooth muscle cells and in some fibroblastic stromal cells. b: Myometrium. Negative control; primary antibody replaced with mouse immunoglobulin. c: Endometrium basalis. Thy 1 is localized mainly to the perivascular region. d: Endometrium basalis. Negative control. e: Endometrium functionalis. Thy 1 immunoreactivity is present in some stromal cells. f: Endometrium functionalis. Negative control. Original magnifications, $\times 400$. Scale bar, $50 \mu \mathrm{m}$.

Primary myometrial and endometrial fibroblast strains were generated and characterized as previously described $^{13}$ (see Materials and Methods for details). Human endometrial and myometrial tissue was cut into pieces of $\sim 1-\mathrm{mm}^{3}$ size. Explants were then placed under glass coverslips in 100-mm diameter Petri dishes, resulting in the outgrowth of fibroblasts from the individual tissues. The cells were tested for expression of markers consistent with fibroblast phenotype. Immunostaining showed the cells to be vimentin-positive (fibroblast mark- er), but negative for CD45 (bone marrow marker), CD34 (endothelial cell marker), $\alpha$ (alpha)-smooth muscle actin (myofibroblasts and smooth muscle cells), and cytokeratin (epithelial cell marker). Confocal staining for Thy 1 demonstrated heterogeneity for this marker in the myometrial and endometrial fibroblast strains. As shown in Figure 2A, the M5 myometrial fibroblast strain contained some cells staining very brightly for Thy 1 , whereas other cells adjacent to them were completely negative for Thy 1 expression. The E7 endometrial strain had a higher per- 

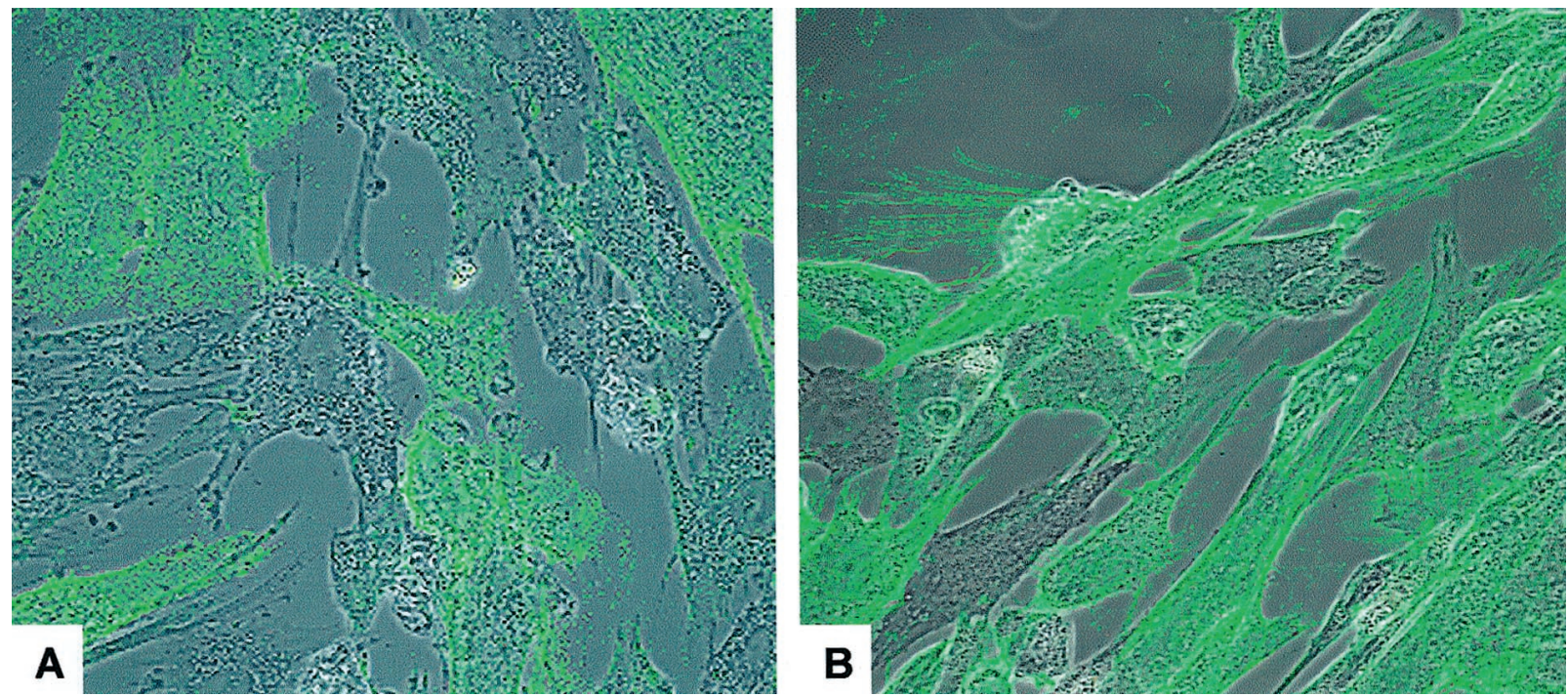

Figure 2. Thy 1 expression by the M5 myometrial fibroblast strain and the E7 endometrial fibroblast strain. Fibroblasts were stained for Thy 1 using the F15-421-5 antibody and analyzed using confocal microscopy. A: Thy 1 expression by the M5 fibroblast strain. Some fibroblasts stain positive for Thy 1 whereas others adjacent to them fail to stain. B: Thy 1 expression by the E7 fibroblast strain. A majority of fibroblasts stain positive for Thy 1 , although there are clearly some Thy $1^{-}$fibroblasts. Original magnifications, $\times 400$.

centage of Thy 1-expressing cells, and thus stained mostly positive for Thy 1 (Figure 2B). Among five different fibroblast strains obtained, Thy 1 expression ranged from 21 to $94 \%$ in myometrial strains and from 27 to $90 \%$ in endometrial strains (Table 1).

\section{Separation of M5 Myometrial Fibroblasts into Thy $1^{+}$and Thy $1^{-}$Subsets}

The myometrial fibroblast strain M5 was chosen for further study, as approximately one half of the cells expressed Thy 1 and it grew relatively well. This made it an ideal candidate for separation into Thy $1^{+}$and Thy $1^{-}$ subsets. Initial analysis using flow cytometry profiles showed that the M5 myometrial fibroblasts were heterogeneous for the surface marker Thy 1, where $41 \%$ of the fibroblasts were Thy $1^{+}$. To separate the cells into Thy 1 subsets, the parental M5 strain was first sorted based on their Thy 1 expression by fluorescence-activated cell sorting, as described in Materials and Methods. Further sorting using three to four rounds of magnetic bead selection resulted in two fibroblast subsets, where one was

Table 1. Diversity of Thy 1 Expression in Human Myometrial and Endometrial Fibroblasts

\begin{tabular}{cccc}
\hline $\begin{array}{c}\text { Myometrial } \\
\text { strain }\end{array}$ & $\%$ Thy $1^{+}$ & $\begin{array}{c}\text { Endometrial } \\
\text { strain }\end{array}$ & $\%$ Thy $1^{+}$ \\
\hline M1 & 77 & E6 & 72 \\
M2 & 93 & E7 & 90 \\
M4 & 94 & E8 & 27 \\
M5 & 44 & E9 & 31 \\
M8 & 21 & E10 & 88
\end{tabular}

Myometrial and endometrial human fibroblast strains were stained with an isotype control antibody or with a mouse anti-human Thy 1 antibody (F15-421-5) and analyzed using in situ immunofluorescence, as well as with flow cytometry.
$>99 \%$ Thy $1^{+}$and the other $>99 \%$ Thy $1^{-}$. These subsets displayed differences in morphology. The Thy $1^{+}$ fibroblasts were larger, with more elongated processes, whereas the Thy $1^{-}$fibroblasts were more rounded and spread. Flow cytometry profiles and morphology of the parental fibroblasts and the Thy $1^{+}$and Thy $1^{-}$subsets are shown in Figure 3.

\section{Cytokine Production by M5 Fibroblast Subsets}

The M5 fibroblast strain and the Thy $1^{+}$and Thy $1^{-}$ populations were next evaluated for rate of proliferation, morphology, and cytokine production. The M5 Thy $1^{+}$ fibroblast subpopulation proliferated at a slower rate than did the M5 Thy $1^{-}$fibroblasts (data not shown). Differences were also observed in their morphology, where the M5 Thy $1^{+}$fibroblasts were more spindle-shaped and had more elongated processes, whereas the M5 Thy $1^{-}$ cells were more rounded and spread (Figure 3; D to F). To determine cytokine production, cells were stimulated with IL-1 $\beta$ or IFN- $\gamma$ for 24 or 72 hours, respectively, as described in Materials and Methods. Supernatants were collected from each treatment, and concentrations of IL-6, IL-8, and MCP-1 were determined by enzyme-linked immunosorbent assay (Figure 4; $A$ to $C$ ). Both subsets produced increased amounts of IL- 6 and IL- 8 after IL- $1 \beta$ stimulation, when compared to untreated cells. IFN- $\gamma$ stimulation did not significantly up-regulate IL-6 or IL-8 production in either subpopulation. This supports the concept of fibroblast heterogeneity across tissues, as only the Thy $1^{+}$ fibroblasts were shown to produce IL-6 in the mouse spleen. ${ }^{22}$ Interestingly, only the M5 Thy $1^{+}$subpopulation was capable of increased MCP-1 production after IFN- $\gamma$ and $\mathrm{IL}-1 \beta$ stimulation. IFN- $\gamma$ treatment yielded a 25 -fold increase and IL-1 $\beta$ a 100-fold increase of MCP-1 over un- 

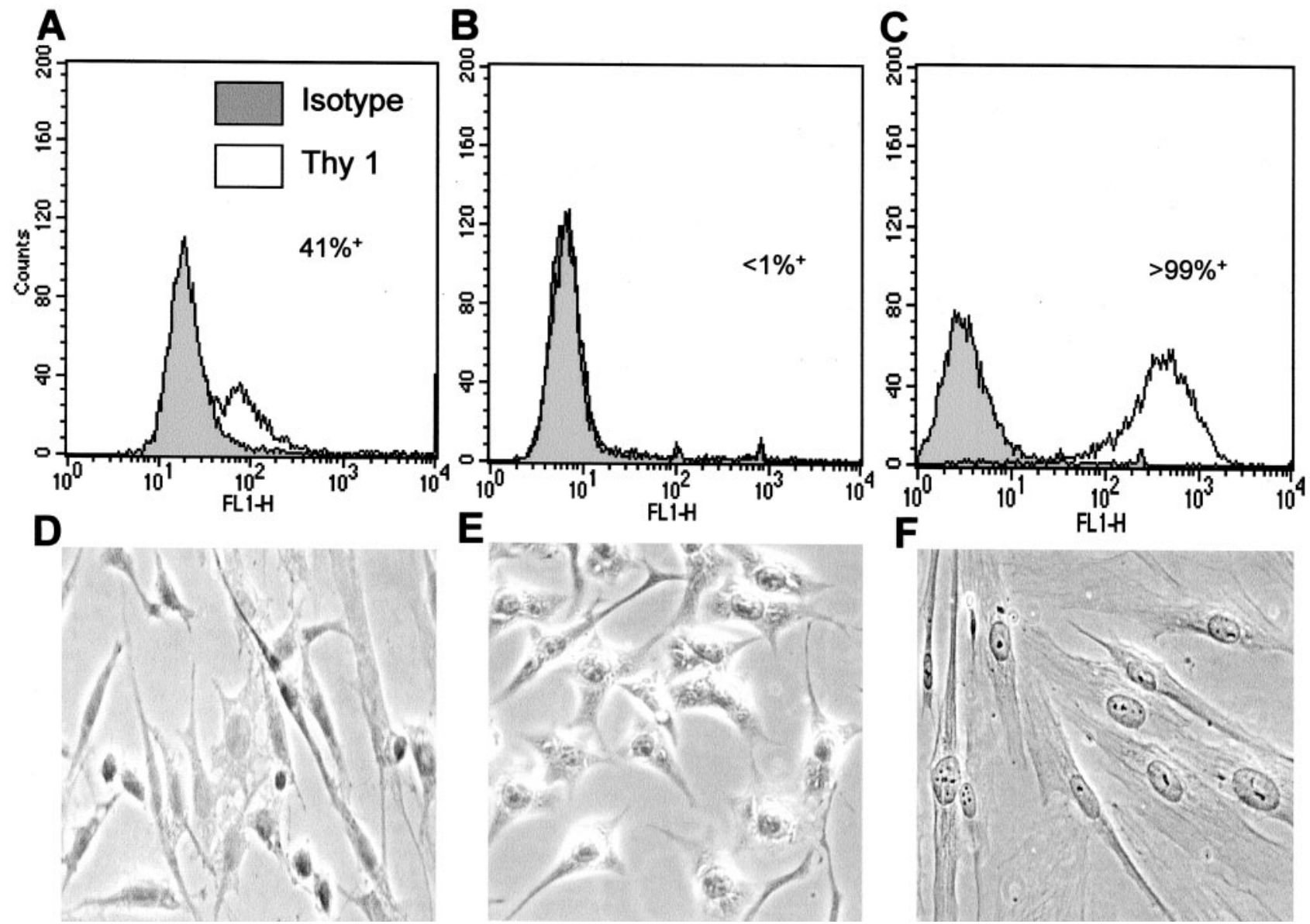

E $\mathrm{FLIH}$

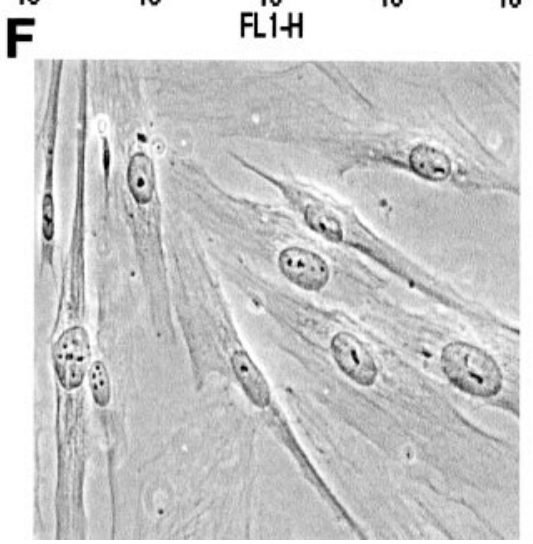

Figure 3. Separation of the M5 fibroblast strain into Thy $1^{+}$and Thy $1^{-}$subsets, A-C: Flow cytometry analysis for Thy 1 expression by M5 parental fibroblasts, showing $41 \%$ of the cells positive for Thy $1(\mathbf{A})$, the M5 Thy $1^{-}$subset, where $<1 \%$ cells were positive for Thy $1(\mathbf{B})$, and the M5 Thy $1^{+}$subset, where $>99 \%$ cells were positive for Thy $1(\mathbf{C})$. Separation into Thy $1^{+}$and Thy $1^{-}$subsets was accomplished using fluorescence-activated cell sorting followed by three to four rounds of magnetic beading as described in Material and Methods. D-F: Phase contrast microscopy (original magnification, $\times 400$ ) indicating morphology of M5 parental (D), M5 Thy $1^{-}(\mathbf{E})$, and M5 Thy $1^{+}$fibroblasts (F). M5 Thy $1^{+}$fibroblasts had more elongated processes whereas the M5 Thy $1^{-}$fibroblasts were more rounded and spread. Fibroblasts were cultured on chamber slides and viewed under phase contrast on a light/phase microscope connected to a digital camera. Original magnification, $\times 400$.

treated cells. M5 Thy $1^{-}$fibroblasts did not induce higher levels of MCP-1 with cytokine stimulation.

\section{M5 Thy $1^{+}$but Not Thy 1 Fibroblasts Up- Regulate CD40 Surface Expression}

We previously reported that myometrial fibroblasts upregulated CD40 expression after IFN- $\gamma$ treatment. ${ }^{13}$ It was of great interest to determine whether the capability to up-regulate CD40 was characteristic of only one of the myometrial fibroblast subsets. Interestingly, only the M5 Thy $1^{+}$subpopulation could up-regulate CD40 expression. As shown in Figure 5A, stimulation with IFN- $\gamma$ for 72 hours or IL-1 $\beta$ for 24 hours induced a substantial upregulation of CD40 of 28 and $26 \%$, respectively. M5 Thy $1^{+}$fibroblasts did not significantly express CD40 at a basal level, with only $2 \%$ of the cells staining positive for surface CD40. The M5 Thy $1^{-}$subpopulation did not considerably up-regulate CD40 expression after either IFN- $\gamma$ or IL- $1 \beta$ stimulation ( $<2 \%$ ) (Figure $5 \mathrm{~B}$ ).

\section{CD40-CD40L Interaction Induces Increased} IL-6, IL-8, and MCP-1 Production by M5 Thy

\section{$1^{+}$Fibroblasts}

Since we established that only M5 Thy $1^{+}$fibroblasts expressed high levels of CD40 on their surface, we sought to determine whether the CD40 was functional. IFN- $\gamma$ was used to up-regulate CD40 surface expression on M5 Thy $1^{+}$fibroblasts. CD40L was then added to ligate CD40 and thus determine the outcome of CD40CD40L interaction on cytokine and chemokine production by the M5 Thy $1^{+}$subset. As shown in Figure 6A, the combination of IFN- $\gamma$ and CD4OL stimulation gave a 3.5fold increase in IL-6 production over IFN- $\gamma$ or CD40L stimulation alone. Addition of the mouse anti-human CD40L antibody MK13A4 to cells treated with IFN- $\gamma$ plus CD40L, completely blocked IL-6 production. Levels of IL-6 were sustained when mouse IgG1 was added as a control antibody to IFN- $\gamma$ - and CD40L-treated cells. Treatment with IFN- $\gamma$ together with CD40L, induced a 


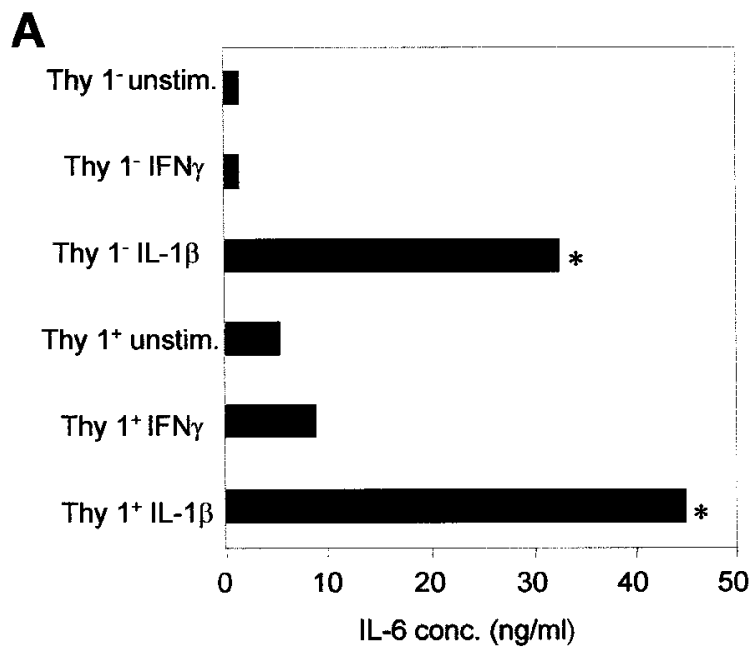

B

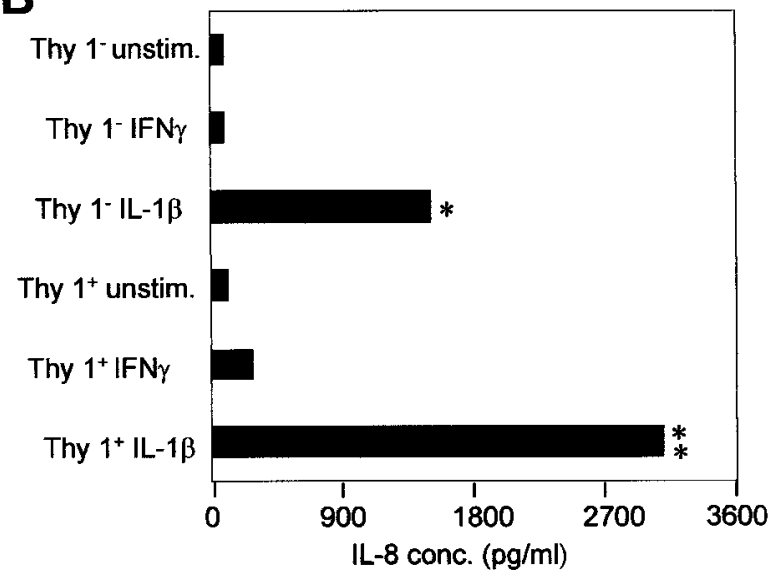

C

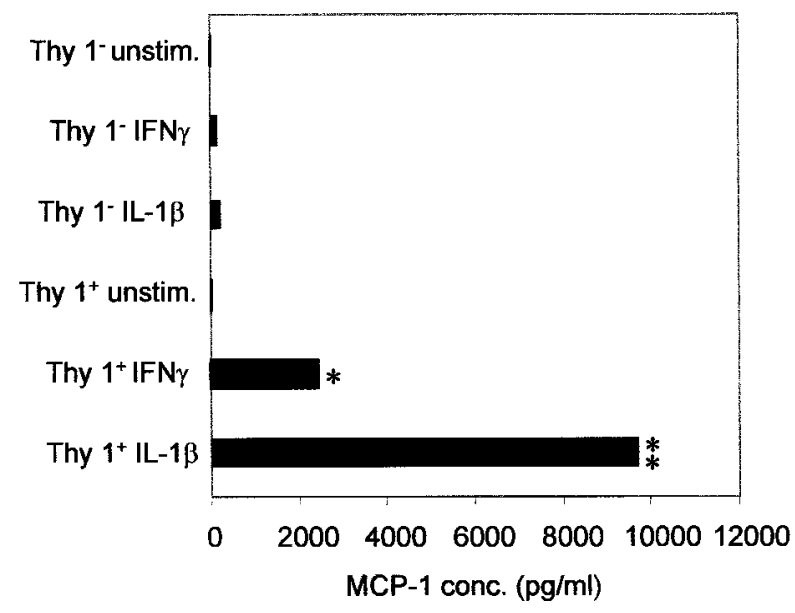

Figure 4. M5 Thy $1^{+}$and Thy $1^{-}$fibroblast subsets synthesize IL-6 and IL-8, but only M5 Thy $1^{+}$fibroblasts produce MCP-1. Cells were serum-starved for 72 hours in $0.5 \%$ FBS and RPMI 1640 and stimulated with IFN- $\gamma$ or IL- $1 \beta$ for the final 24 hours in serum-free media. Supernatants were harvested and analyzed by enzyme-linked immunosorbent assay for IL- 6 and IL- 8 production as described in the Materials and Methods. IL-6 production (A) and IL-8 production (B) by M5 fibroblast subsets. IL- 6 and IL- 8 were produced by both Thy 1 subsets only after IL- $1 \beta$ stimulation. C: MCP- 1 production by M5 fibroblast subpopulations. Only M5 Thy $1^{+}$fibroblasts synthesized significant levels of MCP- 1 in response to IFN- $\gamma$. IL- $1 \beta$ stimulation yielded a further 4.5-fold increase in MCP-1 production. This is a representative of three experiments $\left({ }^{*}, P<0.05 ;{ }^{* *}, P<0.01\right.$ indicates value is statistically significant). sevenfold increase in IL-8 production and a fourfold increase in MCP-1 production in M5 Thy $1^{+}$fibroblasts over stimulations with IFN- $\gamma$ or CD40L alone (Figure 6, B and C). Addition of MK13A4 again reduced IL-8 and MCP-1 to control levels, attributing a specific role of this increase to CD40L and its interaction with CD40. IL-8 and MCP-1 levels in IFN- $\gamma$ - and CD40L-treated cells were not affected by addition of the negative control antibody.

\section{Discussion}

Fibroblasts are dynamic cells whose functions and characteristics vary according to their anatomical location and the environment to which they are exposed. Fibroblast heterogeneity based on Thy 1 expression was first established by this laboratory in the mouse lung and spleen, ${ }^{22,25}$ and by others in the rat lung. ${ }^{29,30}$ Human orbital fibroblasts also seem heterogeneous for Thy 1 expression. ${ }^{17}$ In this article, we report for the first time that fibroblasts from the human endometrium and myometrium are heterogeneous based on bona fide surface marker expression. It is also the first time that human myometrial fibroblasts have been successfully separated into two stable subsets on the basis of a surface marker (Thy 1) and shown to be functionally heterogeneous. We originally proposed that because Thy 1 was successfully used to define unique subpopulations in cells of the hematopoietic lineage ( $B$ and $T$ lymphocytes), it could be also used to delineate functional subsets in structural cells. ${ }^{25}$

Both M5 Thy $1^{+}$and Thy $1^{-}$fibroblast subpopulations up-regulated IL-6 and IL-8 production on stimulation with $\mathrm{IL}-1 \beta$, whereas only the M5 Thy $1^{+}$subset was capable of MCP-1 production. IL-8 is a strong activator and chemoattractant for neutrophils and has important functions in the reproductive tract. ${ }^{1,31}$ Neutrophils release their specific granules (that contain collagenase) when activated and are involved in degrading the collagen fibers in the cervix. ${ }^{32}$ Increased neutrophil accumulation has been observed premenstrually ${ }^{33}$ and in the ripening of the cervix, which is a prerequisite for safe labor. ${ }^{34} \mathrm{IL}-6$ is a proinflammatory cytokine that is shown to participate in cases of premature labor along with $\mathrm{IL}-1 \beta .^{1} \mathrm{MCP}-1$ is a potent chemoattractant for monocytes and is found to increase in the endometrial perivascular region before menstruation. ${ }^{33} \mathrm{MCP}-1$ is up-regulated in the cervix after RU486 treatment and this may cause accumulation of leukocytes contributing to cervical ripening. ${ }^{35} \mathrm{MCP}-1$ also attracts CD4- and CD8-positive T lymphocytes. ${ }^{36}$ So when activated, myometrial Thy $1^{+}$fibroblasts contribute to processes such as cervical ripening, acting as mediators in the initiation of the inflammatory response and neutrophil, monocyte, and T-lymphocyte recruitment via their production of IL-6, IL-8, and MCP-1. If improperly activated, this fibroblast subset could mediate afflictions such as preterm labor and menstrual dysfunction.

It is now clear that the fibroblast is a key sentinel cell that has important functions in fibrosis, wound healing, maintenance of tissue integrity, as well as the regulation of immune responses in various tissues. ${ }^{14,15}$ The CD40- 
A Unstimulated, Thy $1^{+}$

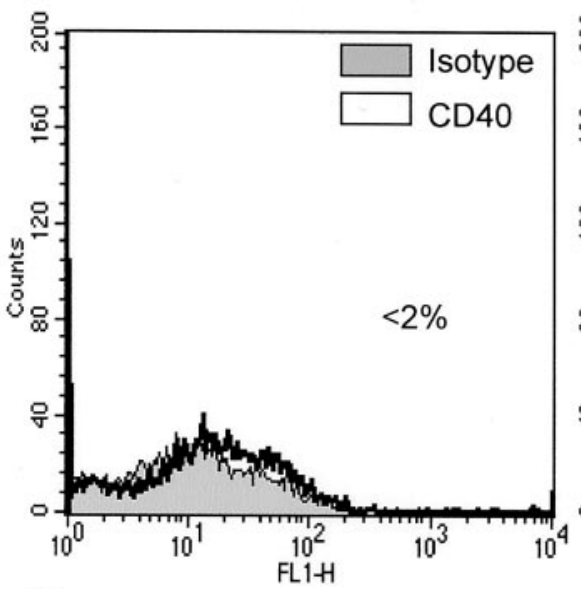

B Unstimulated, Thy 1 -

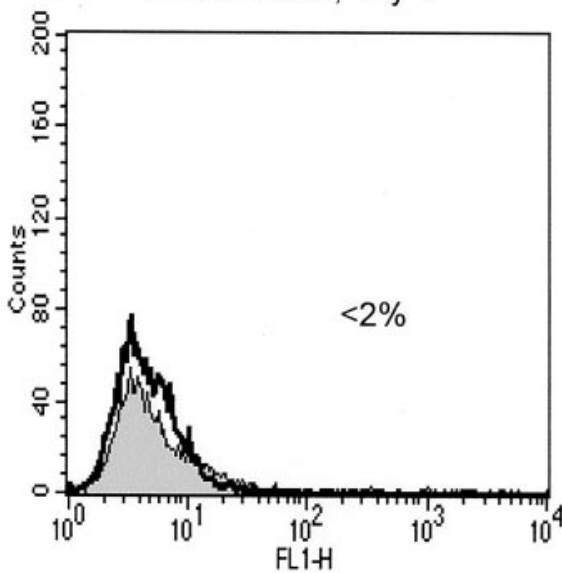

IFN $\gamma$, Thy $1^{+}$

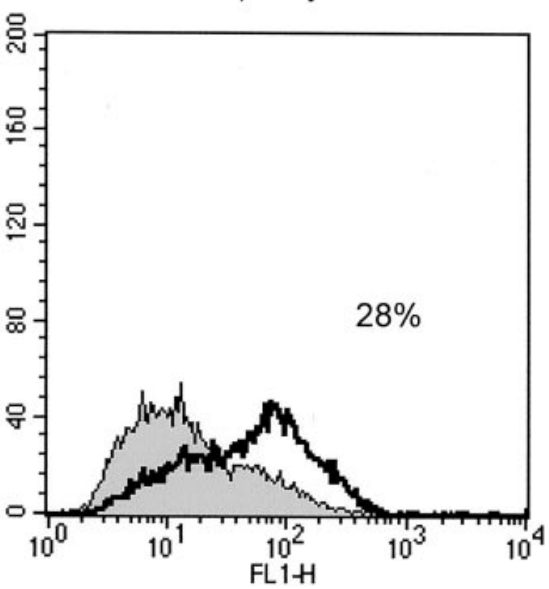

IFN $\gamma$, Thy $1^{-}$

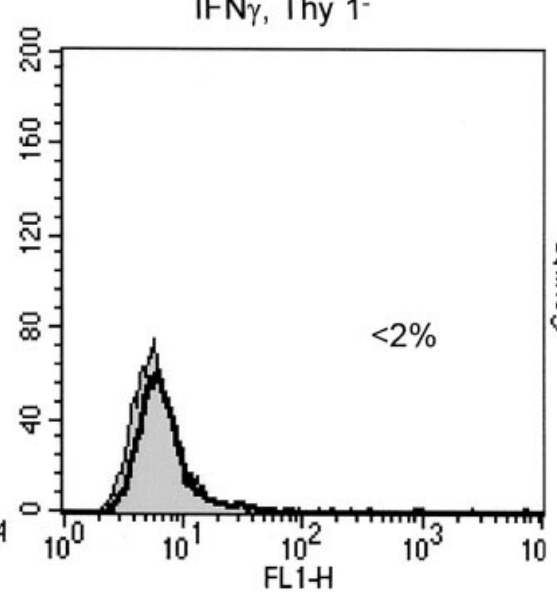

IL-1 $\beta$, Thy $1^{+}$

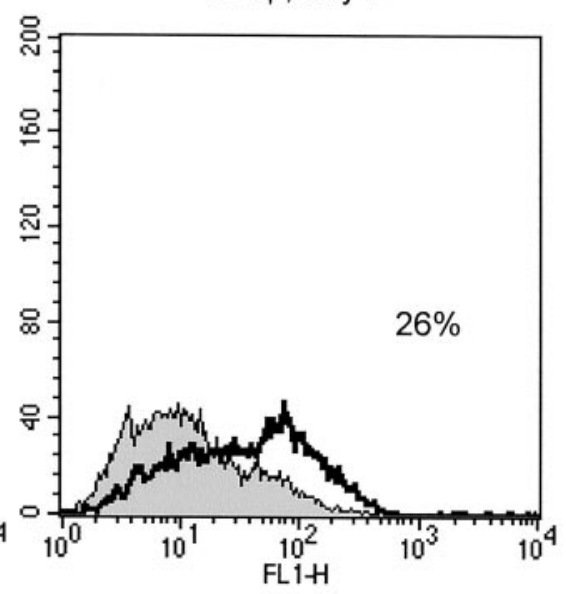

IL-1 $\beta$, Thy 1 -

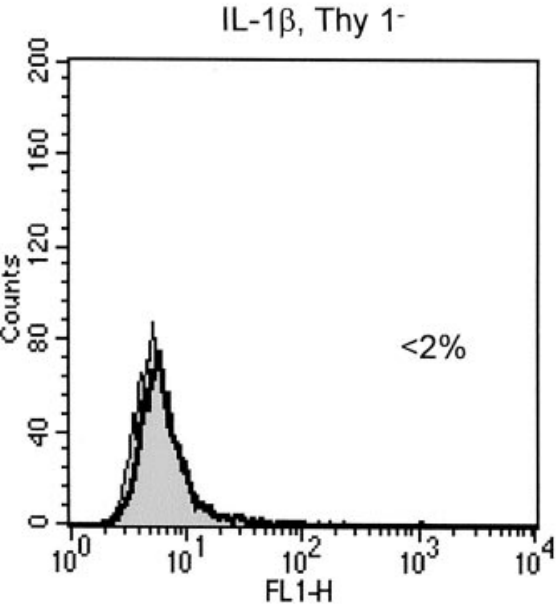

Figure 5. $\mathrm{M} 5$ Thy $1^{+}$fibroblasts express surface CD 40 . Fibroblasts were cultured with or without IFN- $\gamma(500 \mathrm{U} / \mathrm{ml})$ for 72 hours or IL- $1 \beta$ ( 10 ng/ml) for 24 hours. Mouse anti-human CD40 (G28-5) was used as the primary antibody and mouse IgG1 was used as the isotype control. Cells were then incubated with goat anti-mouse Ig-FITC as a secondary antibody. Samples were analyzed on a FACSCalibur. A: CD 40 expression by M5 Thy $1^{+}$fibroblasts. Stimulation with IFN- $\gamma$ led to a $28 \%$ CD 40 surface expression, whereas IL-1 $\beta$ yielded a $26 \%$ CD 40 expression. Unstimulated cells only expressed minimal CD 40 ( $<2 \%$ ). B: CD 40 expression by M5 Thy $1^{-}$fibroblasts. IFN- $\gamma$ and IL- $1 \beta$ stimulation did not up-regulate CD40 expression in the M5 Thy $1^{-}$subset (CD 40 expression, $<2 \%$ ). This figure is representative of three experiments.

CD40L interaction is not only crucial in signaling among cells of the immune system, but is also important for different inflammatory responses of nonhematopoietic cell types. ${ }^{37,38}$ Human orbital fibroblasts were activated through CD40 engagement to produce IL-6 and IL-8. ${ }^{9}$ Human lung fibroblasts activated through the CD40CD40L pathway, showed up-regulated cyclooxygenase-2 expression and prostaglandin $\mathrm{E}_{2}$ production. ${ }^{39}$ Furthermore, human fibroblasts from various tissues and pathological settings are shown to express CD40, suggesting direct interaction with recruited inflammatory cells established through cell adhesion. ${ }^{40}$ Fibroblasts of the human female reproductive system were reported to express CD40 after treatment with IFN- $\gamma .{ }^{13}$ We have established in this report that only the M5 Thy $1^{+}$myometrial fibroblast subpopulation is capable of up-regulating expression of CD40 with IFN- $\gamma$ or IL- $1 \beta$ stimulation, and that CD40 engagement led to increased levels of IL-6, IL-8, and MCP-1. This result designates the myometrial Thy $1^{+}$ fibroblast as a significant player in inflammatory responses involving CD40L-bearing cells such as activated
T cells, eosinophils, ${ }^{7}$ mast cells, ${ }^{6}$ macrophages, ${ }^{41} \mathrm{NK}$ cells, ${ }^{42}$ and activated platelets. $^{8} \mathrm{~T}$ cells and macrophages infiltrate the myometrium at the onset of labor and mast cells exist at higher levels in the nonpregnant state. ${ }^{2}$ Besides interaction via CD40 and CD40L, it is possible that recruited $T$ cells further activate and stimulate chemokine production by Thy $1^{+}$myometrial fibroblasts via their production of IFN- $\gamma$ (Th1 CD4 lymphocytes) at the site of inflammation. In addition, activated macrophages and neutrophils infiltrating the myometrium are a rich source of proinflammatory mediators including IL-1. These proinflammatory mediators are associated with preterm labor and are responsible for inducing the cellular arm of the immune response, which can be detrimental to successful pregnancy. ${ }^{43}$ Alternatively, Th2 CD4 lymphocytes produce cytokines that sustain the humoral arm of the immune response, and support pregnancy. Furthermore, at times of infection and bleeding, activated platelets and their $\mathrm{CD} 40 \mathrm{~L}^{44}$ may assist myometrial Thy $1^{+}$ fibroblasts in playing a role in wound-healing processes in reproductive tissues. 
A
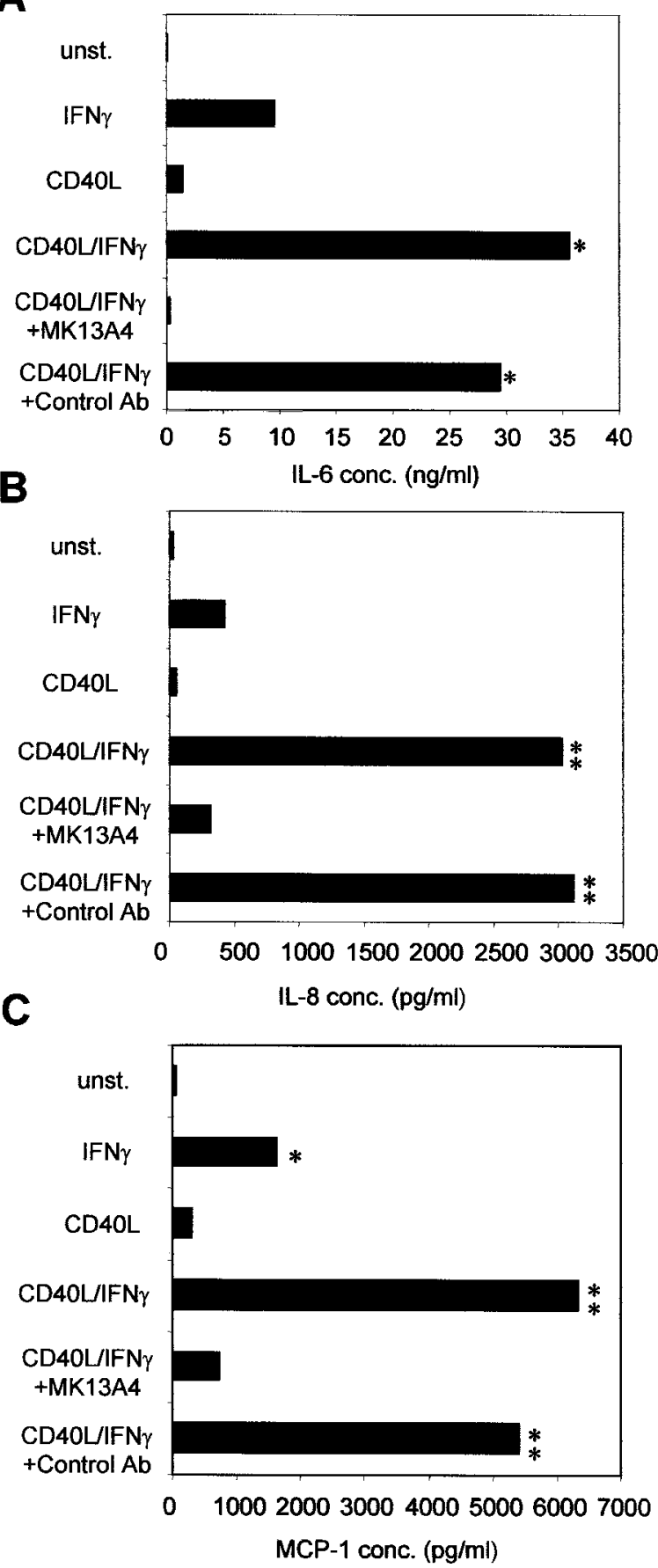

Figure 6. CD 40-CD 40L interaction induces increased IL-6, IL-8, and MCP-1 production by M5 Thy $1^{+}$fibroblasts. Fibroblasts were serum-starved for 72 hours in fresh media containing $0.5 \%$ FBS with or without IFN- $\gamma(500 \mathrm{U} / \mathrm{ml})$. The treatments used for the final 24 hours were: no stimulation, IFN- $\gamma$ alone $(500 \mathrm{U} / \mathrm{ml}), \mathrm{CD} 40 \mathrm{~L}$ alone, or IFN- $\gamma$ together with CD40L. The anti-CD40L antibody MK13A4 $(2.5 \mu \mathrm{g} / \mathrm{ml})$ was used the final 24 hours on fibroblasts stimulated with IFN- $\gamma$ and CD40L. Mouse IgG1 was added at $2.5 \mu \mathrm{g} / \mathrm{ml}$ as a control antibody. Supernatants were harvested and tested for IL-6, IL-8, and MCP-1 production by enzyme-linked immunosorbent assay. A: IL-6 production. IFN- $\gamma$ and CD40L stimulation gave a 3.5-fold increase in IL- 6 production over IFN- $\gamma$ or CD40L stimulation alone. Addition of MK13A4 (anti-CD40L antibody) to cells treated with IFN- $\gamma$ plus CD 40L, completely blocked IL-6 production. B: IL- 8 production. Treatment with IFN- $\gamma$ together with CD40L induced a sevenfold increase in IL-8 production that is blocked by MK13A4. C: $\mathrm{MCP}-1$ production. Addition of IFN- $\gamma$ with CD40L yielded a fourfold increase in MCP-1 production, which is inhibited by MK13A4. Addition of control antibody did not affect induced IL-6, IL-8, or MCP-1 levels (*, $P<$ $0.05 ; * *, P<0.01$ indicates value is statistically significant).
The heterogeneity in Thy 1 expression among myometrial and endometrial fibroblasts from different specimens indicates diverse profiles of Thy $1^{+}$fibroblasts among patients. This could be an indication of the susceptibility of a patient to preterm labor by untimely inflammation or to dysfunctions of the reproductive tract such as menorrhagia. Local distribution of Thy 1 in the reproductive tract also varies within the tissue. For example, in the endometrium, the basalis shows Thy 1 to be restricted to the perivascular cells, whereas in the functionalis Thy 1 expression is widely distributed among stromal cells. The localization of Thy 1 around vessels further ensures the possibility of Thy $1^{+}$fibroblasts coming into contact with infiltrating immune cells. It was recently reported that a natural human Thy 1 ligand is expressed on monocytes and polymorphonuclear cells. ${ }^{45}$ Interaction between Thy 1 and the putative Thy 1 ligand was shown to be involved in the cell adhesion of polymorphonuclear cells (mostly neutrophils) and monocytes to fibroblasts and endothelial cells expressing Thy $1 .{ }^{45}$ Furthermore, Thy 1 is highly expressed in the uterus in new blood vessels formed after pregnancy. ${ }^{46}$ This supports our observations and the hypothesis that the Thy $1^{+}$fibroblasts and Thy 1 localization around blood vessels in the endometrium are important in recruiting and interacting with immune cells. Angiogenesis is critical during pregnancy. Thy $1^{+}$fibroblasts recruit monocytes via production of $\mathrm{MCP}-1$, and these become tissue macrophages that are a source of proinflammatory cytokines and are associated with angiogenesis. ${ }^{46}$ Furthermore, immune cells ( $T$ and $B$ cells) are localized particularly in the basal region, so Thy $1^{+}$ fibroblasts may be involved in their presence. ${ }^{47}$

We conclude that Thy $1^{+}$and Thy $1^{-}$fibroblast subsets in the human female myometrium are unique, dynamic cell populations that act differentially as mediators in controlling normal functions and dysfunctions in the female reproductive tract. Both Thy $1^{+}$and Thy $1^{-}$subsets produced IL-6 and IL-8, whereas only the Thy $1^{+}$ subset was capable of CD40 up-regulation and MCP-1 production. The identification and characterization of distinct fibroblast subpopulations in the human reproductive tract that have unique profiles suggests the importance of considering alternate roles of fibroblast subpopulations in reproductive tract processes associated with inflammation and wound healing. It is likely that each fibroblast subpopulation contributes differently to the development of inflammation by their secretion of soluble mediators that orchestrate the immune response. A delineation of the role of each subpopulation in the reproductive system may provide insight to the development of treatments for preterm labor and reproductive tract disorders, such as menstrual dysfunction.

\section{Acknowledgment}

We thank Dr. M. Kehry (Boehringer Ingelheim, Ridgefield, CT) for providing the mouse anti-human CD40L antibody. 


\section{References}

1. Kelly RW: Pregnancy maintenance and parturition: the role of prostaglandin in manipulating the immune and inflammatory response. Endocr Rev 1994, 15:684-706

2. Thomson AJ, Telfer JF, Young A, Campbell S, Stewart CJ, Cameron IT, Greer IA, Norman JE: Leukocytes infiltrate the myometrium during human parturition: further evidence that labour is an inflammatory process. Hum Reprod 1999, 14:229-236

3. Stamenkovic I, Clark EA, Seed B: A B-lymphocyte activation molecule related to the nerve growth factor receptor and induced by cytokines in carcinomas. EMBO J 1989, 8:1403-1410

4. Hollenbaugh D, Mischel-Petty N, Edwards CP, Simon JC, Denfeld RW, Kiener PA, Aruffo A: Expression of functional CD40 by vascular endothelial cells. J Exp Med 1995, 182:33-40

5. Gaspari AA, Sempowski GD, Chess P, Gish J, Phipps RP: Human epidermal keratinocytes are induced to secrete interleukin-6 and co-stimulate T lymphocyte proliferation by a CD40-dependent mechanism. Eur J Immunol 1996, 26:1371-1377

6. Gauchat JF, Aubry JP, Mazzei G, Life P, Jomotte T, Elson G, Bonnefoy JY: Human CD40-ligand: molecular cloning, cellular distribution and regulation of expression by factors controlling IgE production. FEBS Lett 1993, 315:259-266

7. Gauchat JF, Henchoz S, Fattah D, Mazzei G, Aubry JP, Jomotte T, Dash L, Page K, Solari R, Aldebert D, Capron M, Dahinden C, Bonnejoy JY: CD40 ligand is functionally expressed on human eosinophils. Eur J Immunol 1995, 25:863-865

8. Henn V, Slupsky JR, Grafe M, Anagnostopoulos I, Forster R, MullerBerghaus G, Kroczek RA: CD40 ligand on activated platelets triggers an inflammatory reaction of endothelial cells. Nature 1998, 391:591594

9. Sempowski GD, Rozenblit J, Smith TJ, Phipps RP: Human orbital fibroblasts are activated through CD40 to induce proinflammatory cytokine production. Am J Physiol 1998, 274:C707-C714

10. Fries KM, Sempowski GD, Gaspari AA, Blieden T, Looney RJ, Phipps RP: CD40 expression by human fibroblasts. Clin Immunol Immunopathol 1995, 77:42-51

11. Sempowski GD, Chess PR, Moretti AJ, Padilla J, Phipps RP, Blieden TM: CD40 mediated activation of gingival and periodontal ligament fibroblasts. J Periodontol 1997, 68:284-292

12. Sempowski GD, Chess PR, Phipps RP: CD40 is a functional activation antigen and B7-independent T cell costimulatory molecule on normal human lung fibroblasts. J Immunol 1997, 158:4670-4677

13. King AE, Kelly RW, Critchley HO, Malmstrom A, Sennstrom M, Phipps RP: CD40 expression in uterine tissues: a key regulator of cytokine expression by fibroblasts. J Clin Endocrinol Metab 2001, 86:405-412

14. Phipps RP, Penney DP, Keng P, Silvera M, Harkins S, Derdak S: Immune functions of subpopulations of lung fibroblasts. Immunol Res 1990, 9:275-286

15. Smith RS, Smith TJ, Blieden TM, Phipps RP: Fibroblasts as sentinel cells. Synthesis of chemokines and regulation of inflammation. Am J Pathol 1997, 151:317-322

16. Junqueira LC, Zugaib M, Montes GS, Toledo OM, Krisztan RM, Shigihara KM: Morphologic and histochemical evidence for the occurrence of collagenolysis and for the role of neutrophilic polymorphonuclear leukocytes during cervical dilation. Am J Obstet Gynecol 1980, 138:273-281

17. Smith TJ, Sempowski GD, Wang HS, Del Vecchio PJ, Lippe SD, Phipps RP: Evidence for cellular heterogeneity in primary cultures of human orbital fibroblasts. J Clin Endocrinol Metab 1995, 80:26202625

18. Schneider EL, Mitsui Y, Au KS, Shorr SS: Tissue-specific differences in cultured human diploid fibroblasts. Exp Cell Res 1977, 108:1-6

19. Botstein GR, Sherer GK, Leroy EC: Fibroblast selection in scleroderma. An alternative model of fibrosis. Arthritis Rheum 1982, 25: 189-195

20. Korn JH: Substrain heterogeneity in prostaglandin E2 synthesis of human dermal fibroblasts. Differences in prostaglandin E2 synthetic capacity of substrains are not stimulus-restricted. Arthritis Rheum 1985, 28:315-322

21. Mariotti A, Cuchens M, Hassell T: Vital cell sorting of estrogensensitive human gingival fibroblast subpopulations. J Dental Res 1990, 69:A293

22. Borrello MA, Phipps RP: Differential Thy-1 expression by splenic fibroblasts defines functionally distinct subsets. Cell Immunol 1996, 173:198-206

23. Sempowski GD, Beckmann MP, Derdak S, Phipps RP: Subsets of murine lung fibroblasts express membrane-bound and soluble IL-4 receptors. Role of IL-4 in enhancing fibroblast proliferation and collagen synthesis. J Immunol 1994, 152:3606-3614

24. Noyes RW, Hertig AT, Rock J: Dating the endometrial biopsy. Am J Obstet Gynecol 1975, 122:262-263

25. Phipps RP, Penney DP, Keng P, Quill H, Paxhia A, Derdak S, Felch ME: Characterization of two major populations of lung fibroblasts: distinguishing morphology and discordant display of Thy 1 and class II MHC. Am J Respir Cell Mol Biol 1989, 1:65-74

26. Smith TJ, Sempowski GD, Berenson CS, Cao HJ, Wang HS, Phipps RP: Human thyroid fibroblasts exhibit a distinctive phenotype in culture: characteristic ganglioside profile and functional CD40 expression. Endocrinology 1997, 138:5576-5588

27. Silvera MR, Sempowski GD, Phipps RP: Expression of TGF-beta isoforms by Thy-1+ and Thy-1- pulmonary fibroblast subsets: evidence for TGF-beta as a regulator of IL-1-dependent stimulation of IL-6. Lymphokine Cytokine Res 1994, 13:277-285

28. Saalbach A, Wetzig T, Haustein UF, Anderegg U: Detection of human soluble Thy- 1 in serum by ELISA. Fibroblasts and activated endothelial cells are a possible source of soluble Thy- 1 in serum. Cell Tissue Res 1999, 298:307-315

29. McIntosh JC, Hagood JS, Richardson TL, Simecka JW: Thy1 (+) and $(-)$ lung fibrosis subpopulations in LEW and F344 rats. Eur Respir $\mathrm{J}$ 1994, 7:2131-2138

30. Hagood JS, Miller PJ, Lasky JA, Tousson A, Guo B, Fuller GM, McIntosh JC: Differential expression of platelet-derived growth factoralpha receptor by Thy-1(-) and Thy-1(+) lung fibroblasts. Am J Physiol 1999, 277:L218-L224

31. Kelly RW, Illingworth P, Baldie G, Leask R, Brouwer S, Calder AA: Progesterone control of interleukin-8 production in endometrium and chorio-decidual cells underlines the role of the neutrophil in menstruation and parturition. Hum Reprod 1994, 9:253-258

32. Osmers R, Rath W, Adelmann-Grill BC, Fittkow C, Kuloczik M, Szeverenyi $\mathrm{M}$, Tschesche $\mathrm{H}$, Kuhn W: Origin of cervical collagenase during parturition. Am J Obstet Gynecol 1992, 166:1455-1460

33. Jones RL, Kelly RW, Critchley HO: Chemokine and cyclooxygenase-2 expression in human endometrium coincides with leukocyte accumulation. Hum Reprod 1997, 12:1300-1306

34. Lange AP, Secher NJ, Westergaard JG, Skovgard I: Prelabor evaluation of inducibility. Obstet Gynecol 1982, 60:137-147

35. Denison FC, Riley SC, Elliott CL, Kelly RW, Calder AA, Critchley HO: The effect of mifepristone administration on leukocyte populations, matrix metalloproteinases and inflammatory mediators in the first trimester cervix. Mol Hum Reprod 2000, 6:541-548

36. Carr MW, Roth SJ, Luther E, Rose SS, Springer TA: Monocyte chemoattractant protein 1 acts as a T-lymphocyte chemoattractant. Proc Natl Acad Sci USA 1994, 91:3652-3656

37. van Kooten C, Banchereau J: Functional role of CD40 and its ligand. Int Arch Allergy Immunol 1997, 113:393-399

38. van Kooten C, Banchereau J: Functions of CD40 on B cells, dendritic cells and other cells. Curr Opin Immunol 1997, 9:330-337

39. Zhang Y, Cao HJ, Graf B, Meekins H, Smith TJ, Phipps RP: CD40 engagement up-regulates cyclooxygenase-2 expression and prostaglandin E2 production in human lung fibroblasts. J Immunol 1998, 160:1053-1057

40. Brouty-Boye D, Pottin-Clemenceau C, Doucet C, Jasmin C, Azzarone B: Chemokines and CD40 expression in human fibroblasts. Eur J Immunol 2000, 30:914-919

41. Mach F, Schonbeck U, Sukhova GK, Bourcier T, Bonnefoy JY, Pober JS, Libby P: Functional CD40 ligand is expressed on human vascular endothelial cells, smooth muscle cells, and macrophages: implications for CD40-CD40 ligand signaling in atherosclerosis. Proc Natl Acad Sci USA 1997, 94:1931-1936

42. Carbone E, Ruggiero G, Terrazzano G, Palomba C, Manzo C, Fontana S, Spits H, Karre K, Zappacosta S: A new mechanism of NK cell cytotoxicity activation: the CD40-CD40 ligand interaction. J Exp Med 1997, 185:2053-2060

43. Wegmann TG, Lin H, Guilbert L, Mosmann TR: Bidirectional cytokine interactions in the maternal-fetal relationship: is successful pregnancy a TH2 phenomenon? Immunol Today 1993, 14:353-356

44. Phipps RP: Atherosclerosis: the emerging role of inflammation and 
the CD40-CD40 ligand system. Proc Natl Acad Sci USA 2000, 97: 6930-6932

45. Saalbach A, Haustein UF, Anderegg U: A ligand of human thy- 1 is localized on polymorphonuclear leukocytes and monocytes and mediates the binding to activated thy-1-positive microvascular endothelial cells and fibroblasts. J Invest Dermatol 2000, 115:882-888
46. Lee WS, Jain MK, Arkonac BM, Zhang D, Shaw SY, Kashiki S, Maemura K, Lee SL, Hollenberg NK, Lee ME, Haber E: Thy-1, a novel marker for angiogenesis upregulated by inflammatory cytokines. Circ Res 1998, 82:845-851

47. Loke YW, King A, Burrows TD: Decidua in human implantation. Hum Reprod 1995, 2(Suppl. 10):14-21 